\title{
Hydro-mechanical paths within unsaturated compacted soil framed through water retention surfaces
}

\author{
Benjamin Pelizzari ${ }^{1,2, a}$, Apostolia Kontogianni ${ }^{1,2}$, Simon Salager $^{1,2}$ and Olivier Ple Pl, $^{3}$ \\ ${ }^{1}$ Univ. Grenoble Alpes, 3SR, F-38000 Grenoble, France \\ ${ }^{2}$ CNRS, 3SR, F-38000 Grenoble, France \\ ${ }^{3}$ Univ. Savoie Mont Blanc, LOCIE, F-73000 Chambéry, France \\ ${ }^{4}$ CNRS, LOCIE, F-73000 Chambéry, France
}

\begin{abstract}
Compaction is a key issue of modern earthworks... From sustainable development, a need arise of using materials for compaction under given conditions that would normally be avoid due to unpredictable pathologies. The application of compaction on fine grained soils, without a change of gravimetric water content, lead to very important modifications of the void ratio and hence suction. Therefore the hydro-mechanical behaviour of fine grained soil need to be rendered around three variables: suction, void ratio, saturation degree or water content. The barring capacity of the soil is assessed through Penetrometers (In-situ manual penetrometer, CBR) in order to assess gains through compaction. The three states variables are then assessed for in situ and frame through water retention surfaces, realized from Proctor tests, in which compaction effect and path could be described.
\end{abstract}

\section{Introduction}

Earthwork relies in great part on compaction for the realisation of key infrastructures. As simple as compaction seems, its methods might differ greatly depending on the region it is executed. More other, compaction of fine grained soils without use of hydraulic binder is greatly disregarded, even not recommended by standards. This study as part of the French ANR project TerreDurable [1] aims to better understand the behaviour of marl under compaction with different energies, water content and compactors. The effect on the three main state variable of the soil are then assessed (density, suction, degree of saturation). In this regard, full scale on site compaction tests are studied from samples, and then are compared to laboratory compaction tests (Proctor) [2].

\section{Full scale compaction tests}

\subsection{Studied Material}

The fined grained soil studied is marl extracted from the earthworks of the A304. This Highway aims to link Belgium to Charleville-Mézières in France. The materials are defined as A2 by French standard regulation (GTR) [3]. Its undersize at $85 \mu \mathrm{m}$ is more than $35 \%$ and its plasticity index is 24 (Tab. 1). Their geological origin lies in the Pliensbachian and/or the Toarcian. Some materials will be tested undisturbed; those aim to simulate supports soils. Others will be reworked, either on site as they would for earthworks, either with standard compaction tests.

Table 1. Reference Values for the marl

\begin{tabular}{|c|c|}
\hline Propriety & Values \\
\hline Liquidity limit & $46 \%$ \\
\hline Plasticity limit & $22 \%$ \\
\hline Plasticity index & $24 \%$ \\
\hline Natural water content & $20 \%$ \\
\hline Grains density & $2.665 \mathrm{gcm}^{-3}$ \\
\hline
\end{tabular}

\subsection{Layout and goals}

The parameters and their variations (Tab.2) are defined aiming to see compaction pathologies. Hence the need arise to use dry and wet materials that respectively increases the possibilities for lamination and wave effect. Tamping shoe on rammer tends to overcome those two pathologies while the flat roller facilitates them (with help from the vibration). The two other parameter variations are here to assess the effects of the use of pulvimixer (mostly not regarded if the soil is non-treated) and over-compaction energy.

The test is carried out in three layers. The two first layer are used as a compaction base for the third layer that will receive on one side a normal compaction ( 2 times back

\footnotetext{
a Corresponding author: benjamin.pelizzari@3sr-grenoble.fr
} 
and forth), and the other side over-compaction (6 times back and forth). The water contents are attained by natural drying (scarification/sun/wind) and wetting by injection. Each set of parameters on the compaction site represented a compacted bloc of soil of $15 \mathrm{~m}$ length, $10 \mathrm{~m}$ wide (4 compactor rammers width) and three layers about $30 \mathrm{~cm}$. Figure 1 shows test realization. After compaction, different measurements are carried out on the layer (manual dynamic penetrometer, gammadensimeter). Samples are then made for laboratory test by force driving a tube in the compacted soil and extracting it.

Table 2. Parameter variation for on-site experimentations

\begin{tabular}{|c|c|}
\hline Parameters & Variations \\
\hline $\begin{array}{c}\text { Compaction } \\
\text { Energy }\end{array}$ & Increase of the number of passes \\
\hline Compactor Type & Flat roller and tamping roller \\
\hline Material Humidity & $\begin{array}{c}\text { Medium, dry side and wet side } \\
\text { of normal Proctor }\end{array}$ \\
\hline Material grinding & mixed and raw material \\
\hline
\end{tabular}

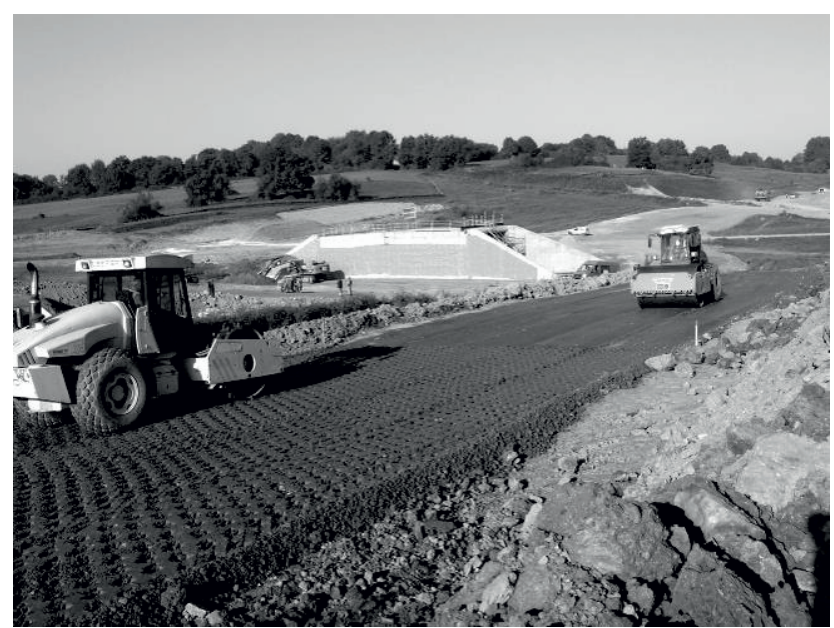

Figure 1. In situ compaction test construction.

\subsection{Results of the full scale test}

The effect of the gridding is not visible in any of cases studied. It seems that the clods were not hard enough to remain uncrushed. This is corroborated by penetrometer measurements executed in the soil depot before compaction. As well, the compactor type is indecisive. Though Wave effect appears on one on the wet bloc compacted when using flat roller. On the opposite, tamping roller does not trigger it. The most interesting results come from over-compaction. A loss in mechanical resistance is observed on the wet side with the increase in number of passes while the dry material gained in strength. The medium humidity soil showed transition behaviour with gain and losses depending of the compaction set.

\subsection{Core samples analysis}

\subsubsection{Methodology}

The core samples are opened and then three slices of $\sim 1 \mathrm{~cm}$ are cut at the top part and at a $20 \mathrm{~cm}$ depth. The goal is to try to compare the evolution of density, saturation and suction at two depth of the compaction layer, densification being stronger at the top of the layer. The slices are wrapped with filter papers for suction measurements. After the filter weighing, density and saturation degree are obtained through hydrostatic weighing of the slices in a non-wetting liquid. The water content is finally obtained by stove drying.

\subsubsection{Results}

Before analysing the results, on site experimental details must be discussed. The material was extracted at water content close to the optimum proctor, though, to obtain wet material, water injection was needed. A good homogeneity of the material after such a process is hard to attain in such huge proportions. Looking at the measurements of the samples, this heterogeneity shows, and the results have to be studied accordingly.
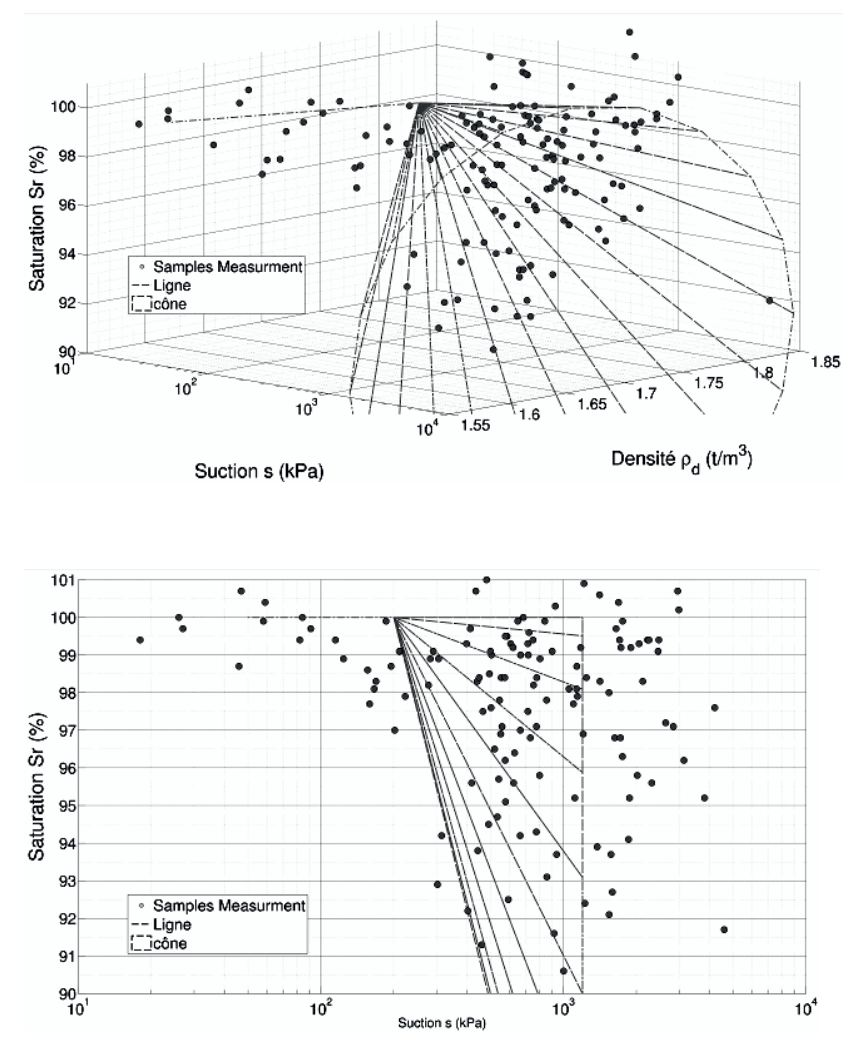

Figure 2. Core sample point spread, 3D and 2D projection.

The compaction test points seem to be spread widely but some sense can make from the process even though the heterogeneity. The global results are presented in figure 2. First, the graph above should not be treated as a retention diagram but as a compaction results. Hence no drying or wetting path appears. The points for low 
suction and density present higher degrees of saturation. A minimal air entry value of the material then appears for suction around $200 \mathrm{kPa}$. After that point, there is a spread of the data in a circumferential surface. All those points belong to volume in which could be drawn series of water retention surfaces each depending of its own density. The aim is then to try to frame those data using laboratory testing, and generating water retentions surfaces for the materials. As a reminder, it should be noted that the samples are all very close to saturation $(\mathrm{Sr}>90 \%)$.

Table 3 present the evolution of the suction and the density regarding compaction test depending of the preparation water content.

Table 3. Density and suction variation with compaction

\begin{tabular}{|c|c|c|c|c|}
\hline \multirow{2}{*}{$\begin{array}{c}\text { Initial } \\
\text { position }\end{array}$} & \multicolumn{2}{|c|}{ Normal compaction } & \multicolumn{2}{c|}{ Over-compaction } \\
\cline { 2 - 5 } & Suction & Density & Suction & Density \\
\hline Wet side & 1263 & 1.710 & 832 & 1.650 \\
\hline Optimum & 1231 & 1.740 & 658 & 1.770 \\
\hline Dry side & 728 & 1.725 & 180 & 1.730 \\
\hline
\end{tabular}

An element that arises from this table is that, whatever the water content, density is increased by compaction except on the wet side. On the opposite, suction values change drastically. The dryer the material, the less effect over-compaction will have on suction. On wet material the losses of suction are important. Increase in compaction energy does not change the suction after compaction on the dry side, but does affect it on the wet side of the proctor [4]. These sudden changes in suction and density may explain the mechanical response of the soils as it depends mostly on those two values. A loss of suction will lower the strength of the material while higher density will increase it.

\section{Laboratory compaction tests}

\subsection{Experimental layout}

The goal of the lab tests is to frame the results obtained with in situ experimentations regarding the three state variables. Soils taken from the compaction test is stove dried, then sieved under a $5 \mathrm{~mm}$ size, then wetted to different water content and finally compacted in an automatic proctor machine. Before compaction, one week was left to the material to reach homogeneity. Two compaction energies are used: normal and modified, in order to represent the two in situ degrees of compaction. The moulds are lubricated before test. After compaction, left other of the uncompacted soil is placed in an air tight recipient with a filter paper to measure the matric suction before compaction. The Proctors are then submitted to a CBR test. Finally, small samples are extracted from Proctors and are submitted to a drying wetting cycle. Suction is measured thanks to filter papers and a water retention curve for the $100 \%-70 \%$ saturation degree is established. Density and saturation degree are obtained by hydrostatic weighing.

\subsection{Mechanical response}

The mechanical response, observed through CBR tests, showed the same tendencies as the penetrometer on the full scale compaction test. The CBR showed better results for modified proctor on the dry side whereas the resistance was lost on the wet side of the normal Proctor.

Same results were obtained by Sawangsuriya et al. (2000) the elastic part of the mechanical response using bender elements [5]. The hypothesis of the direct dependence of the mechanical response to suction and density is confirmed, whatever the energy or the compaction method, if density and suction are the same for two different samples, the mechanical response will be the same [6]. The increase of energy plays an important role on suction depending on the water content regarding the normal optimum. It is to be noted that those variations of suction appear only when fine grained soils are in sufficient proportions [7].

\subsection{Evolution of suction during compaction}

One of the final elements of this series of test was to compare the suction before and after compaction. Figure 3 presents those values. The variations in suction are nearly negligible when on the dry side. The wetter the material, the more it will lose suction by the effect of compaction. Obviously, it confirms that increased compaction energy might lower even more the suction than normal compaction. Hence bearing capacity is lost, and might lead to disorders.

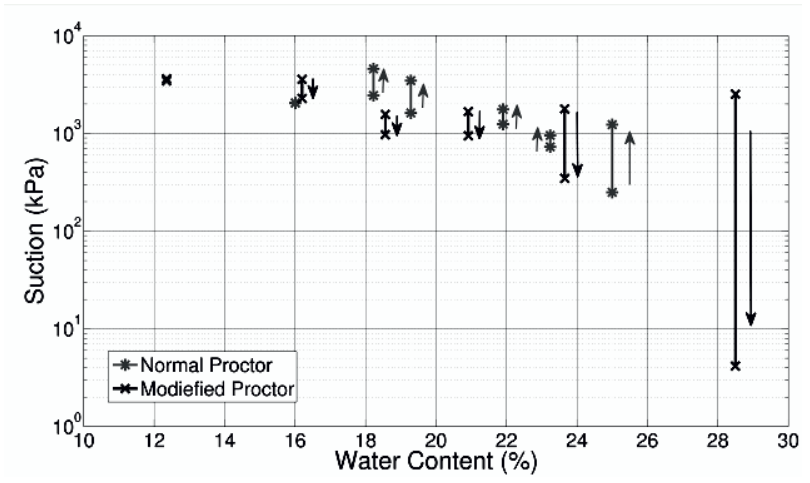

Figure 3. Paths followed during compaction 


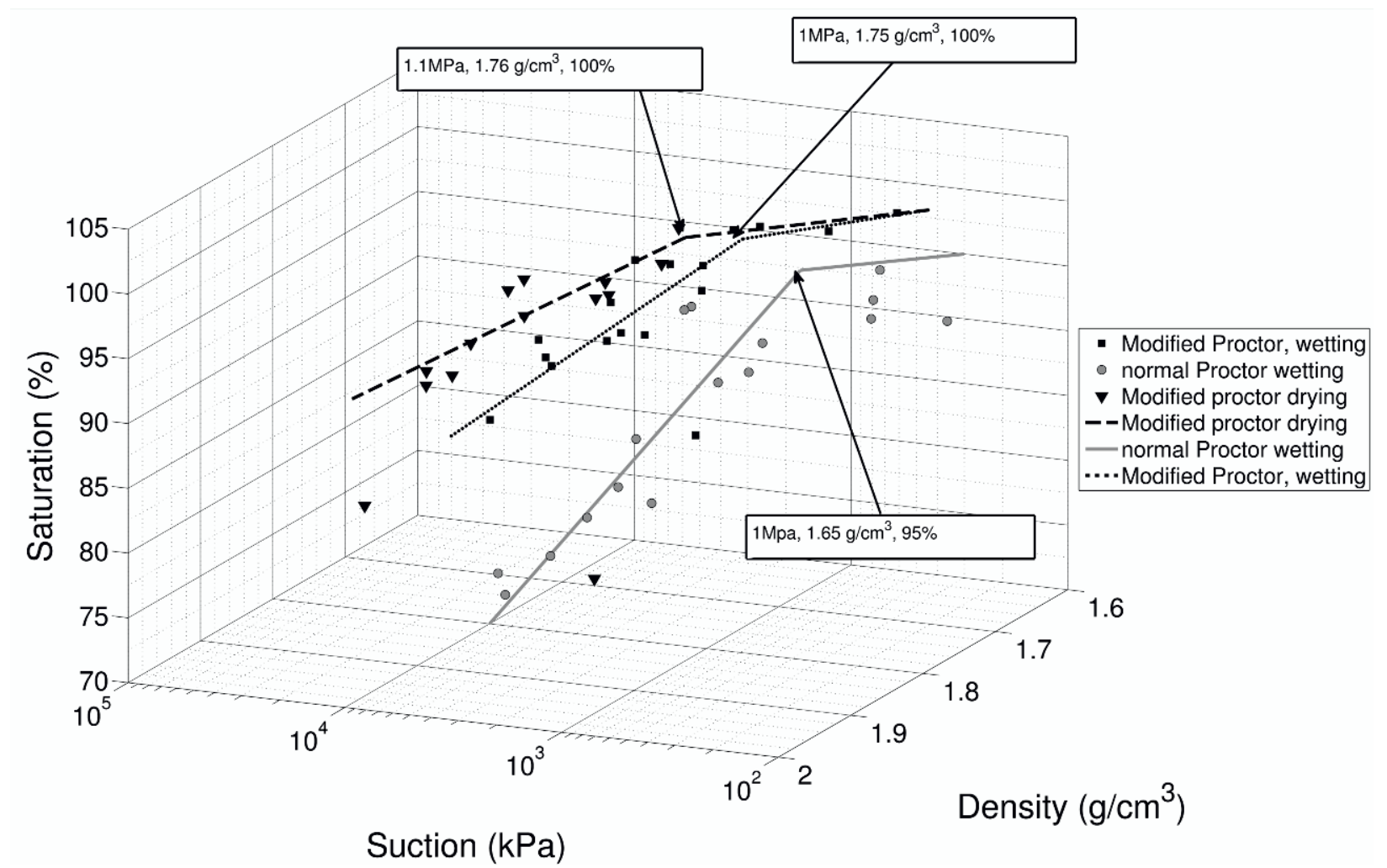

Figure 4. Water retention curves for normal and modified Proctor.

\subsection{Retention curves}

After extraction of soil samples from the different Proctors, water retention curves where realized and are shown in figure 4 . The graph presents three curves. Two represents the drying and wetting curves of the modified Proctor. The third one is the wetting curve of the normal Proctor. The curves are defined and fitted as bilinear. Since in situ compaction gives saturation degrees above $90 \%$, the retention curves are only processed to values around $70 \%$. It is possible to establish a retention surface between the pair of wetting and drying curves [8].

Each compaction energy gives a totally different water retention curve, mostly due to the variation of the density of compaction. It seems that the water retention curves relatively well align. Whatever the water content before compaction, only one wetting and one drying curve appears per water content and energy pair when several would have been expected (one per initial density). The estimated air entry point fairly evolves with the compaction energy, giving a value of $1.1 \mathrm{MPa}$ for the modified proctor, and a relatively lower value under $1 \mathrm{MPa}$ for the normal proctor. This type of result is corroborated by other studies $[9,10]$ find in the literature. The main difference between the two curves comes from their respective densities. The density of the material will define its skeleton structure and shape (pore size) and will directly influence the suction and saturation at air entry. The water retention at lower suction should not be affected by compaction [11].

\section{Crossing analysis}

\subsection{Proctor graph with core samples data}

Showing all points issued from in situ test samples and the proctor points obtained in laboratory with the stove drying, an anomaly arises in figure 5 . It seems that all the points of the compaction test present a density greater than the modified proctor, with a greater saturation degree. This result is unusual for earthworks, though appears sometimes on site with fine grained soils, mostly with clays and marl. Looking at the drying method, it seems that the stove dries the material too much at $105^{\circ} \mathrm{c}$, removing part of the tightly adsorbed water and tends to offset the proctor curve on the dry side. It should be noted that the French standard for the Proctor recommend $50^{\circ} \mathrm{C}$. For the same gridding of the material, same optimum density is obtained but with a different optimum water content. The modification of the grinding before compaction tends to modify the optimum density. Proctor tests prepared with natural drying shows a better harmony with the in situ points of compaction. 


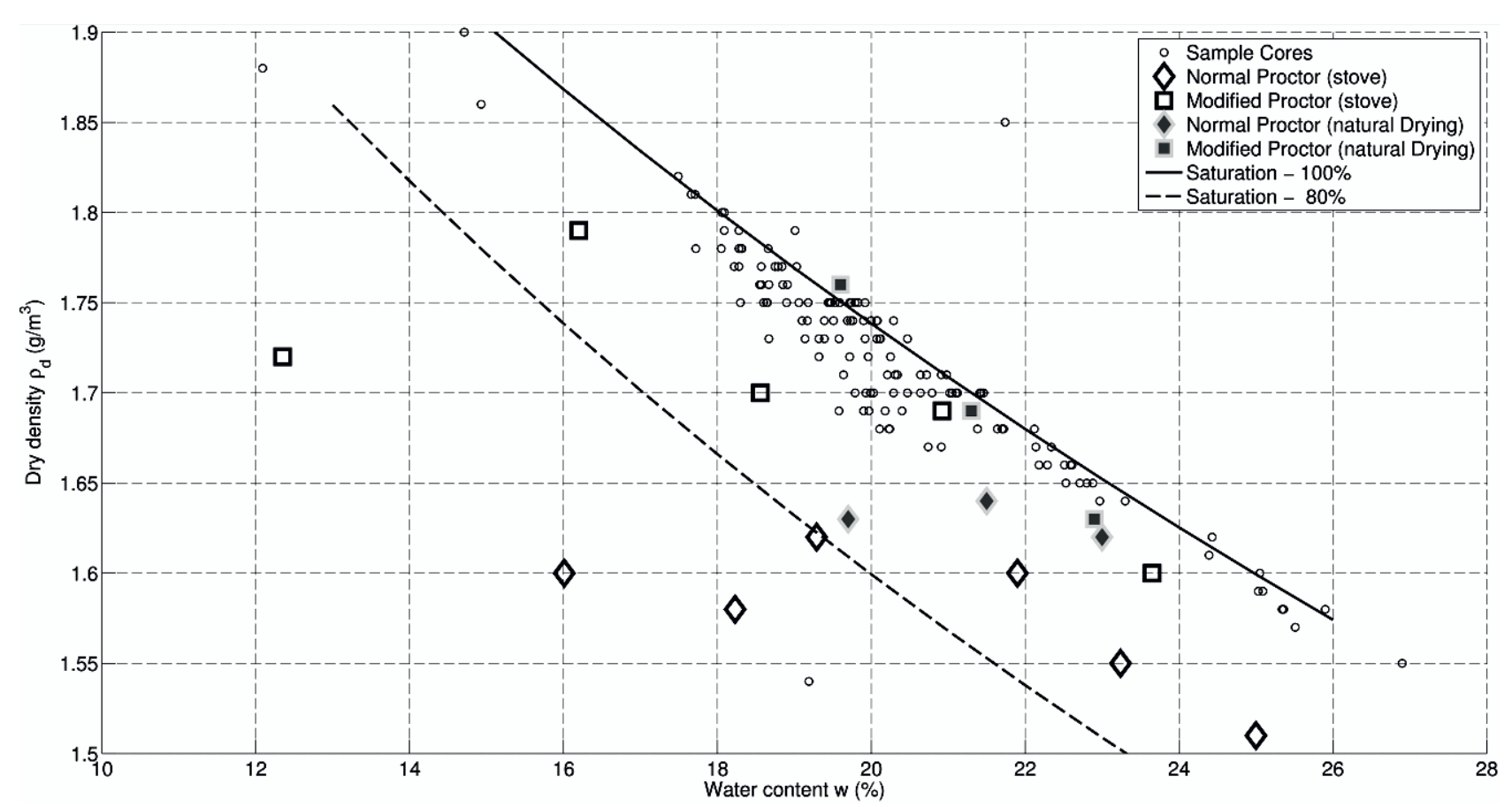

Figure 5. Proctor domain with sample values and Proctors

\subsection{Core samples point saturation framing}

Even if the proctor is offset, the comparison between the points seems to give a good parallel. Taking the retention lines drawn in figure 4, and placing them on the samples points in figure 6 , a good framing of the samples is obtained. The compaction points are nearly all contained in between the retention curves. With several energies, a batch of curves could be drawn and a retention surface could be extracted. The air entry of the modified proctor aligns well with drier points of the compaction test.

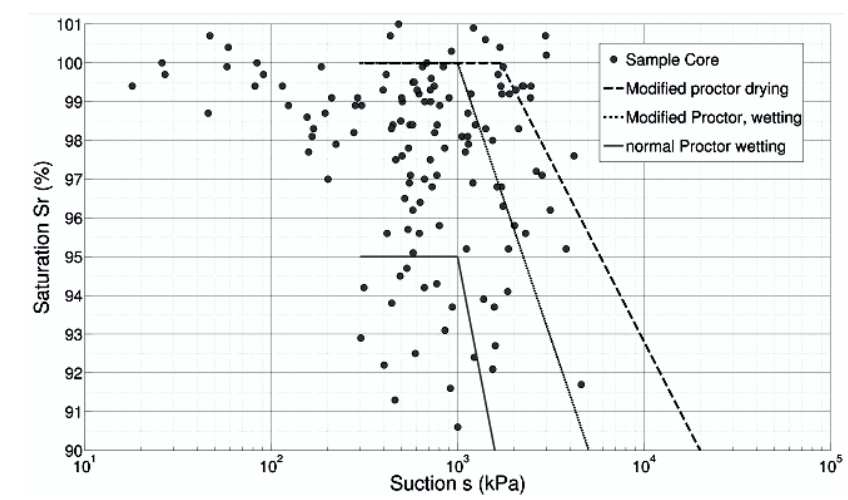

Figure 6 Evolution of suction during compaction

Nearly all the points of the in situ test are under the drying curve of the modified Proctor. Hence this retention curve gives the maximum air entry obtainable while compaction occurs on our material at water content between $14 \%$ and $26 \%$ of water content. This is confirmed as the points have reach $100 \%$ of saturation and any more energy applied would not be able to increase the density. More other, the lower limit given by the wetting curve of the normal proctor gives a fair lower limit; even if it seems that a lower energy would be able to provide a lower curve.

\section{Conclusions}

The in situ compaction test showed impact of the compaction energy used during earthworks. Important losses of mechanical strength depending of the water content are visible. These results were then confirmed by laboratory experimentations.

Globally over compaction, for fine grained soils, reduces suction on the wet side of the proctor while rest mostly unchanged for dryer material. At the same time, density is roughly maintained or increased whatever the water content. These effects lead to a competition between loss of resistance by suction lowering and a gain of resistance by densification of the material that will define the mechanical response of the soil.

Moreover, it is possible to frame the in situ compaction results in a water retention volume, defined from water retention curve from proctor at different energies. The results allow assessing the evolution of the air entry in the material depending of its compaction degree.

The test carried out showed also that the representativity of laboratory tests can be questioned on work site. The material dried using a stove showed a 
modification of its proctor curve manifested by an offset of the optimum water content on the dry side by a nonnegligible 3 points but with same optimum density, as the grinding was the same for both.

\section{Acknowledgements}

The authors would like to thank Vinci Construction Terrassement for financing our laboratory in the context of the French ANR project TerreDurable

\section{References}

1. L. Boutonnier, J. Monnet, J.-M. Fleureau, J.-J. Fry, B. Loret, J.-P. Magnan, O. Plé, K. Wong, TerreDurable Terrassements économiques écologiques et durables, Agence Nationale de la Recherche - Programme Bâtiments et Villes durables (2012) [ANR-11-VILD-0004]

2. Camp S., Plé O. et Gourc J.P. Proposed protocol for characterizing a clay layer subjected to bending Geotechnical Testing Journal : 32, 3 (2009).

3. LCPC et SETRA. Guide des Terrassements Routiers, Réalisation des remblais et des couches de forme, Guide technique (GTR). T. 1, 2. Ministère de l'équipement du logement et des transports français, (2000).

4. E. Romero, A. Gens, A. Lloret. Water permeability, water retention and microstructure of unsaturated compacted boom clay. Engineering Geology 54 p.117-127 (1999).
5. S. Tripathy, E. C. Leong, H. Rahardjo. Suction of compacted residual soils, Unsaturated Soils : Experimental Studies, Springer, p.111-122 (2005).

6. V. Sivakumar, J. Wheeler. Influence of compaction procedure on the mechanical behaviour of an unsaturated compacted clay Part 1: Wetting and isotropic compression. Géotechnique 50, 4 p.359-368 (2000)

7. A. Sawangsuriya, T. B. Edil, P. J. Bosscher. Modulus-Suction-Moisture Relationship for compacted soils in postcompaction state. Journal of Geotechnical and geoenvironmental engineering 135, 1, p.1390-1403 (2009).

8 S. Salager, M. S. El Youssoufi, C. Saix. Definition and experimental determination of a soil-water retention surface, Can. Geotech. J. 47 p.609-622 (2010).

9. S. K. Vanaplly, D. G. Fredlund, D. E. Pufahl Influence of soil structure and stress history on the soil-water characteristics of a compacted till. Géotechnique 49, 2 p143-159 (1997).

10. F A. M. Marinho, M. M. Stuermer, The Influence of the compaction energy on the SWCC of a residual soil, Advances In Unsaturated Geotechnics p.125141 (2012).

11. S. Frydman, R. Baker. Theoretical soil-water characteristic curves based on adsorption, cavitation, and a double porosity model. International journal of Geomechanics 96 p.250-257 (2009). 